\title{
Influência do ambiente no conteúdo calórico e na condição fisiológica de duas espécies migradoras de peixes neotropicais
}

\author{
Laryssa H. R. Pazianotoํㅜ, Maíra L. S. Souza², Ana Lúcia Veronezzi ${ }^{3} \&$ Evanilde Benedito ${ }^{1,2,3,4}$
}

\begin{abstract}
1. Programa de Pós-Graduação em Ecologia de Ecossistemas Aquáticos Continentais, Universidade Estadual de Maringá, Av. Colombo, 5790, Blocos G90 e H90, Maringá, PR, Brasil. (lary.pazianoto@hotmail.com) 2. Curso de Ciências Biológicas, Departamento de Biologia, Universidade Estadual de Maringá, Av. Colombo, 5790, Blocos H78, sala 5, 87020-900 Maringá, PR, Brasil. (lary.pazianoto@hotmail.com) Programa de Pós-Graduação em Biologia Comparada, Universidade Estadual de Maringá, Av. Colombo, 5790, Blocos G80, sala 201, 87020-900 Maringá, PR, Brasil.

4. Núcleo de Pesquisas em Ictiologia, Limnologia e Aquicultura (Nupelia), Universidade Estadual de Maringá, Av. Colombo, 5790, Blocos G90 e H90, 87020-900 Maringá, PR, Brasil.
\end{abstract}

\begin{abstract}
Influence of the environment on caloric content and physiological condition of two species of migratory Neotropical fishes. This paper aims to establish the energy density and condition factor of two fish species, one iliophagous and another piscivorous, in the Upper Paraná River floodplain, and possible seasonal and temporal differences. Samples were taken from June 2010 to March 2011, and muscle samples were extracted from the region next to the dorsal fin, then rinsed in distilled water, and dried at $60^{\circ} \mathrm{C}$. Subsequently, they were macerated and burned in a calorimetric pump. Regarding the subsystems, the energy density and the condition factor showed significant differences only for Prochilodus lineatus (Valenciennes, 1837), with higher values at the Ivinhema subsystem. In relation to the type of environment, no significant differences were detected for any of the species, for both energy density and condition factor. As for the hydrological cycle, significant differences were found for the energy density and the condition factor of Pseudoplatystoma corruscans (Spix \& Agassiz, 1829), with greater values in September and March, respectively. Although the analysis for Prochilodus lineatus had not evidenced significant differences, we observed higher values of energy density and condition factor in June, pointing out a possible influence of the hydrological cycle on the reproductive period of these fish species. In conclusion, the energy density and condition factor of fish can suffer seasonal and temporal variations, according to the environment and the examined hydrological cycle.
\end{abstract}

KEYWORDS. Pseudoplatystoma corruscans, Prochilodus lineatus, energy density, condition factor, floodplain.

RESUMO. O presente estudo teve por objetivo determinar o conteúdo calórico e fator de condição de uma espécie de peixe iliófaga e outra piscívora, na planície de inundação do alto rio Paraná, e suas possíveis diferenças sazonais e temporais. As espécies foram amostradas entre junho de 2010 e março de 2011 e, dos indivíduos que se apresentaram no estádio de desenvolvimento gonadal em repouso, extraíram-se músculos próximos à nadadeira dorsal, os quais foram enxaguados em água destilada, e secos a $60^{\circ} \mathrm{C}$. Posteriormente, foram macerados e queimados em bomba calorimétrica. Em relação aos subsistemas, a densidade energética e o fator de condição apresentaram diferenças significativas apenas para Prochilodus lineatus (Valenciennes, 1837), com os maiores valores constatados no subsistema Ivinhema para as duas análises. Nas análises por tipo de ambiente não foram encontradas diferenças significativas para nenhuma das espécies, tanto para densidade energética, quanto para fator de condição. Em relação ao ciclo hidrológico, foram registradas diferenças significativas para a densidade energética e fator de condição de Pseudoplatystoma corruscans (Spix \& Agassiz, 1829), com os maiores valores para o mês de setembro e março, respectivamente. Apesar das análises para Prochilodus lineatus não terem apresentado diferenças significativas, constataram-se os maiores valores de densidade energética e fator de condição para o mês de junho, indicando a possível influência do ciclo hidrológico no período reprodutivo das referidas espécies. Assim, conclui-se que a densidade energética e o fator de condição dos peixes podem sofrer variações sazonais e temporais, de acordo com o ambiente analisado e o ciclo hidrológico.

PALAVRAS-CHAVE. Pseudoplatystoma corruscans, Prochilodus lineatus, densidade energética, fator de condição, planície de inundação.

As planícies de inundação apresentam um mosaico de hábitats aquáticos, terrestres e de transição. No ecossistema aquático, diferenças na velocidade da água, profundidade, persistência do ambiente aquático (e.g. lagoas perenes e temporárias) e, principalmente das características físicas e químicas, proporcionam uma grande heterogeneidade ambiental (ThOMAz et al., 1997). Mas, apesar das significativas diferenças entre estes sistemas, no que diz respeito aos seus constituintes físico-químicos e bióticos, mecanismos de interação são observados, quer seja pela unificação do sistema em grandes cheias ou pela comunicação através de uma rede de canais.

Constata-se também uma elevada variabilidade temporal, usualmente associada com as flutuações dos níveis fluviométricos, da qual derivou o conceito de pulso de inundação (JUNK et al., 1989) ou simplesmente pulso, incluindo uma fase seca e outra de cheia (NEIFF, 1990). $\mathrm{Na}$ fase de incremento destes níveis, diferentes biótopos expandem sua superfície e profundidade, originando ligações com outros ambientes. Este mecanismo de conexão é o meio mais usual para que diversas comunidades possam acessar diferentes ambientes localizados na planície, desenvolvendo ali processos de alimentação e reprodução (LOWE-MCCONNELL, 1999). Estas conexões podem implicar também em diferenças na qualidade da água.

Com isso, não se pode deixar de considerar a grande influência causada pelo próprio ambiente e pelo ciclo hidrológico na fisiologia das espécies, já que em regiões tropicais, profundas variações temporais e espaciais de variáveis limnológicas são registradas nos sistemas rios-planícies de inundação (ROBERTo et al., 2009).

Sendo assim, estudos que indiquem a condição fisiológica das espécies, bem como particularidades referentes à alimentação e reprodução, servem como subsídio para o desenvolvimento de medidas de manejo que visem à manutenção da fauna ictiológica. Essa tem se tornado uma preocupação constante, devido à marcante redução dos estoques pesqueiros que se tem observado ao longo dos anos, principalmente quando se 
tratam de espécies migradoras que, geralmente, possuem uma elevada importância econômica e na alimentação da população, como é o caso de Prochilodus lineatus (Valenciennes, 1837) e Pseudoplatystoma corruscans (Spix \& Agassiz, 1829).

Prochilodus lineatus (curimba) apresenta-se amplamente distribuído pela América do Sul (FowleR, 1950), estando entre as dez espécies mais abundantes nos desembarques pesqueiros da porção inferior da planície de inundação do alto rio Paraná (COSTA et al., 2012). Alimenta-se de lodo, algas, perifiton e detritos orgânicos, sendo considerada espécie de regime alimentar especializado, do tipo iliófago (AzEvEDo \& VieIRA, 1938; LeITE et al., 1988; Fugi \& HAHN, 1991).

Pseudoplatystoma corruscans (pintado), por sua vez, é um piscívoro migrador de grande porte que pode alcançar mais de $1,5 \mathrm{~m}$ de comprimento. Entretanto, apesar da elevada importância econômica, a pesca predatória, aliada aos impactos resultantes dos represamentos tem contribuído com a depleção dos estoques (AbilhoA \& Duboc, 2004). Assim como $P$. lineatus, $P$. corruscans também possui ampla distribuição na América do Sul, especialmente nas bacias dos rios Paraná-Paraguai e bacia do rio São Francisco (GrAÇA \& PAVANelLi, 2007).

Em espécies migradoras, o acúmulo de lipídios nos tecidos e o seu consequente incremento calórico são de grande importância para a efetivação dos mecanismos associados à reprodução. Assim, os padrões de reserva e direcionamento de energia no metabolismo são fontes de informações valiosas para a compreensão de sua ecologia (Saldaña \& Venables, 1983). Além disso, a densidade calórica de uma espécie pode influenciar os cálculos do modelo de consumo, produção e eficiência de conversão, os quais são imprescindíveis para o estabelecimento de modelos bioenergéticos e na obtenção de respostas para questões específicas no manejo de recursos naturais (ECONOMIDS et al., 1981).

Sendo assim, o presente estudo objetivou investigar o conteúdo calórico de $P$. lineatus e $P$. corruscans em diferentes locais da planície de inundação do alto rio Paraná, a fim de elucidar possíveis influências de variáveis limnológicas e do ciclo hidrológico sobre o armazenamento de energia muscular e sobre a condição fisiológica geral, partindo da hipótese de que devido seu hábito migrador, não existe variação no conteúdo calórico dessas espécies ao longo da bacia. Os resultados obtidos, além de possibilitarem o estabelecimento de medidas de manejo adequadas à manutenção das espécies, também ilustram os efeitos do ambiente sobre a ecologia energética das mesmas.

\section{MATERIAL E MÉTODOS}

Área de estudo. A área de estudo compreende o trecho de planície de inundação do alto rio Paraná situado abaixo da barragem de Porto Primavera e a montante do reservatório de Itaipu. As coletas trimestrais foram realizadas entre junho de 2010 e março de 2011, em pontos de amostragens de três subsistemas: subsistema

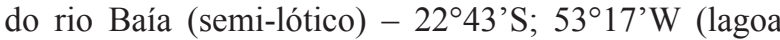
Fechada, lagoa Guaraná, lagoa da Onça, lagoa do Gavião, lagoa Pousada das Garças, canal Baía, canal Curutuba e rio Baía), subsistema do rio Ivinhema (lótico) $-22^{\circ} 47^{\prime} \mathrm{S}$; $53^{\circ} 32^{\prime} \mathrm{W}$ (lagoa Ventura, lagoa dos Patos, lagoa Finado Raimundo, lagoa Peroba, lagoa Sumida e rio Ivinhema) e subsistema do rio Paraná (lótico) - $22^{\circ} 45^{\prime} \mathrm{S}$; $53^{\circ} 15^{\prime} \mathrm{W}$ (lagoa das Garças, canal Cortado e rio Paraná) (Fig. 1).

Segundo Roberto et al. (2009), dos três subsistemas amostrados, o subsistema Paraná apresenta os mais elevados valores médios de transparência da água obtidos através de disco de Secchi $(3 \mathrm{~m})$, enquanto o subsistema Ivinhema e Baía apresentam valores médios inferiores $(0,7 \mathrm{~m}$ e $0,8 \mathrm{~m}$ respectivamente) . Quanto aos nutrientes, destacam-se os baixos valores médios de fósforo total para o subsistema Paraná (12 $\left(\mu \mathrm{g} . \mathrm{L}^{-1}\right)$, em contrapartida aos elevados valores médios registrados para os dois outros subsistemas, de $39 \mu \mathrm{g} . \mathrm{L}^{-1}$ para o subsistema Ivinhema e de $38 \mu \mathrm{g} . \mathrm{L}^{-1}$ para o Baía.

Coleta e análise de dados. As amostragens de $P$. corruscans e $P$. lineatus foram realizadas com redes de espera de malhagens de 3 a $16 \mathrm{~cm}$ entre nós opostos, expostas por 24 horas, e inspecionadas a cada oito horas. Cada exemplar coletado teve determinado seu comprimento padrão $(\mathrm{Ls}$ em $\mathrm{cm}$ ) e peso total (Wt em g), além do sexo e estádio de desenvolvimento gonadal. Os dois últimos parâmetros foram definidos com base nos critérios estabelecidos por VAzzoLER (1996), que para o desenvolvimento gonadal apresenta os seguintes estádios: imaturo, repouso, maturação, maduro e esgotado. Porém, para o presente estudo foram selecionadas apenas espécimes em repouso, o que se justifica pela necessidade de eliminar as variações que ocorrem durante o processo de preparação das gônadas para a reprodução (Tab. I). Exemplares estão depositados na Coleção Ictiológica do Museu do Núcleo de Pesquisas em Limnologia, Ictiologia e Aquicultura (Nupelia) da Universidade Estadual de Maringá - UEM (NUP12692 e NUP13784).

As amostras de músculo de indivíduos de cada espécie foram extraídas da região próxima à inserção da nadadeira dorsal, lavadas em água destilada e incluídas em papel alumínio, rotuladas e congeladas. Posteriormente, as amostras foram secas em estufa de ventilação forçada $\left(60^{\circ} \mathrm{C}\right)$ até obtenção de peso seco constante, maceradas com o auxílio de um moinho de esferas e peneiradas, a fim de se obter um pó fino e homogêneo.

A densidade energética (DE) dessas amostras foi medida em quilocalorias por grama de peso seco $(\mathrm{Kcal} / \mathrm{g}$ PS) e determinada em um calorímetro adiabático.

O estado fisiológico das espécies, avaliado por meio do fator de condição, foi obtido pela fórmula $\mathrm{K}=$ $\mathrm{Wt} / \mathrm{Ls}^{\mathrm{b}}$, sendo b o coeficiente angular da regressão linear entre Wt e Ls (SANTOS et al., 2006). 


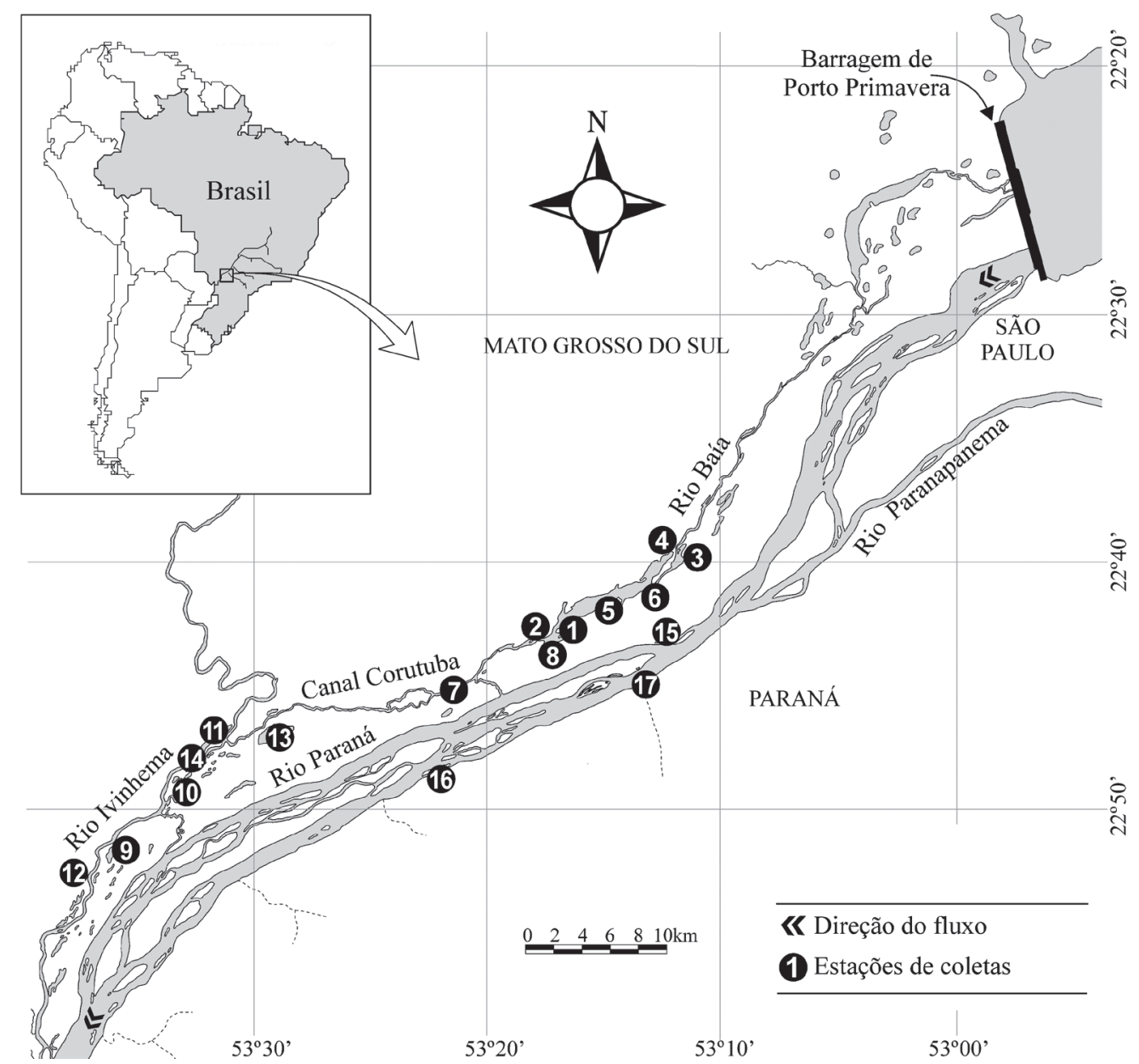

Fig. 1. Locais de amostragem de exemplares de Pseudoplatystoma corruscans (Spix \& Agassiz, 1829) e Prochilodus lineatus (Valenciennes, 1837) na planície de inundação do alto rio Paraná, coletados entre junho de 2010 e março de 2011: 1, Lagoa Fechada; 2, Lagoa Guaraná; 3 , Lagoa da Onça; 4, Lagoa do Gavião; 5, Lagoa Pousada das Garças; 6, Canal Baía; 7, Canal Curutuba; 8, Rio Baía; 9, Lagoa Ventura; 10, Lagoa dos Patos; 11, Lagoa Finado Raimundo; 12, Lagoa Peroba; 13, Lagoa Sumida; 14, Rio Ivinhema; 15, Lagoa das Garças; 16, Canal Cortado; 17, Rio Paraná.

Os valores de $\mathrm{DE}$ e $\mathrm{K}$, determinados para as duas espécies, foram analisados por tipo de ambiente (lagoas abertas, lagoas fechadas, canais e rios), período (meses de coleta) e subsistemas (Ivinhema, Baía e Paraná), considerando-se os valores de mediana e percentis.

Para isso, os ambientes foram agrupados em: lagoas fechadas (lagoa Pousada das Garças, lagoa Ventura e lagoa Fechada), lagoas abertas (lagoa Finado Raimundo, lagoa dos Patos, lagoa Sumida, lagoa do Gavião, lagoa da Onça, lagoa Guaraná, lagoa Peroba, lagoa das Garças), canais (canal Ipoitã, canal Curutuba, canal Cortado e canal Baía) e rios (rio Ivinhema, rio Baía e rio Paraná).

Para as análises sazonais e do ciclo hidrológico, considerou-se os meses de coleta: junho, setembro, dezembro e março, e não as fases do ciclo hidrológico, uma vez que este se mostrou atípico durante o período de coleta, com a cheia ocorrendo apenas em março de 2011, quando o esperado seria em novembro/dezembro de 2010 (Fig. 2) (dados da estação fluviométrica de Porto São José, Paraná, AgÊNCIA Nacional de ÁGUAS, 2011).

Possíveis diferenças entre os valores obtidos para $\mathrm{DE}$ e para $\mathrm{K}$, foram testadas por meio da Análise de Variância não-paramétrica Kruskal-Wallis, com correção de Bonferroni, utilizando o programa STATISTICA 7.0.

\section{RESULTADOS}

A tabela I apresenta o número de exemplares coletados e utilizados para as análises de DE (densidade energética) e K em cada mês de coleta.

Tab. I. Número de indivíduos em repouso utilizados para as análises de densidade energética e fator de condição, por espécie amostrada e sexo (M, macho; F, fêmea), na planície de inundação do alto rio Paraná, entre junho de 2010 e março de 2011.

\begin{tabular}{lcccccccc}
\hline \multirow{2}{*}{ Espécie } & \multicolumn{2}{c}{ Junho 2010 } & \multicolumn{2}{c}{ Setembro 2010 } & \multicolumn{2}{c}{ Dezembro 2010 } & \multicolumn{2}{c}{ Março 2011 } \\
\cline { 2 - 8 } & $\mathrm{M}$ & $\mathrm{F}$ & $\mathrm{M}$ & $\mathrm{F}$ & $\mathrm{M}$ & $\mathrm{F}$ & $\mathrm{M}$ & $\mathrm{F}$ \\
\hline Prochilodus lineatus & 2 & 24 & 0 & 10 & 3 & 9 & 9 & 24 \\
Pseudoplatystoma corruscans & 1 & 7 & 7 & 6 & 1 & 6 & 0 & 2 \\
\hline
\end{tabular}




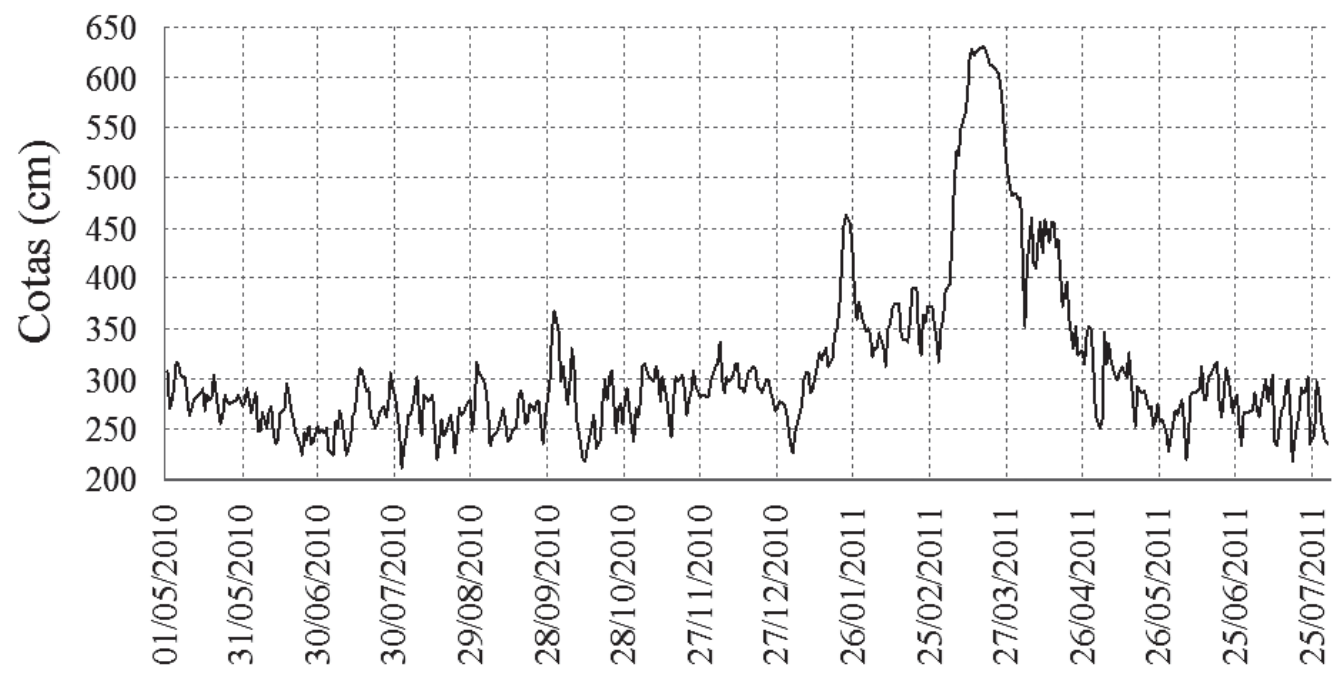

Fig. 2. Cotas fluviométricas para a planície de inundação do alto rio Paraná no período de maio de 2010 a julho de 2011.

Pseudoplatystoma corruscans. Tanto as análises de DE, quanto as de K por subsistema e tipo de ambiente não demonstraram diferenças significativas. Para DE os maiores valores foram verificados no subsistema Paraná e nas lagoas abertas (Figs 3,4) e para $\mathrm{K}$ foram observados no subsistema Ivinhema e nos rios (Figs 5, $6)$.

Acerca da influência sazonal e do ciclo hidrológico, registrou-se diferença significativa para $\mathrm{DE}$, sendo junho significativamente diferente de setembro (Fig. 7). Para os valores de $\mathrm{K}$ também foram verificadas diferenças significativas, sendo junho significativamente diferente de setembro e março, com os maiores valores para este último (Fig. 8).

Prochilodus lineatus. Constatou-se diferenças significativas para DE entre os subsistemas, sendo o Ivinhema significativamente diferente do Baía (Fig. 9). Na análise de DE por tipo de ambiente não foram observadas diferenças significativas, entretanto os maiores valores foram verificados para os rios (Fig.
10). Para as análises de $\mathrm{K}$ também foram registradas diferenças significativas por subsistema, sendo Ivinhema diferente de Baía e Paraná (Fig. 11). Para a citada espécie, os maiores valores foram observados para o subsistema Ivinhema, tanto para as análises de DE quanto para as de $\mathrm{K}$. Quanto ao tipo de ambiente não foram encontradas diferenças significativas para $o$ $\mathrm{K}$, sendo os maiores valores registrados para os canais e lagoas abertas (Fig. 12).

Quanto ao período, não foram reveladas diferenças significativas nem para as análises de DE, nem para as de $\mathrm{K}$, com os maiores valores verificados para o mês de junho nas duas situações (Figs 13, 14).

\section{DISCUSSÃO}

Os valores de DE (densidade energética) e K para $P$. corruscans não apresentaram diferenças significativas entre os subsistemas avaliados e, além disso, os maiores valores de cada um desses parâmetros não ocorreram
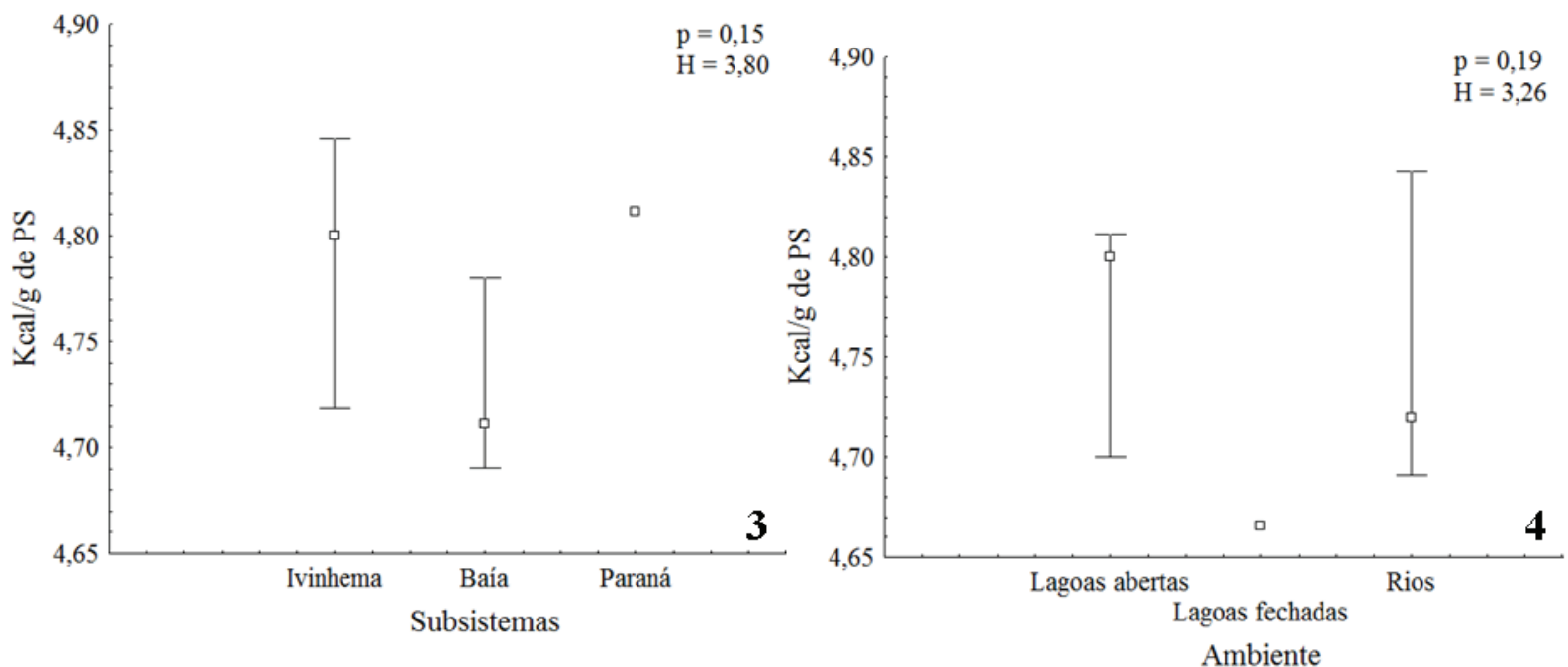

Figs 3, 4. Mediana ( $\square$ ) e percentis (barras verticais) da densidade energética por subsistema (3) e tipo de ambiente (4) para Pseudoplatystoma corruscans (Spix \& Agassiz, 1829) na planície de inundação do alto rio Paraná, entre junho de 2010 e março de 2011. 

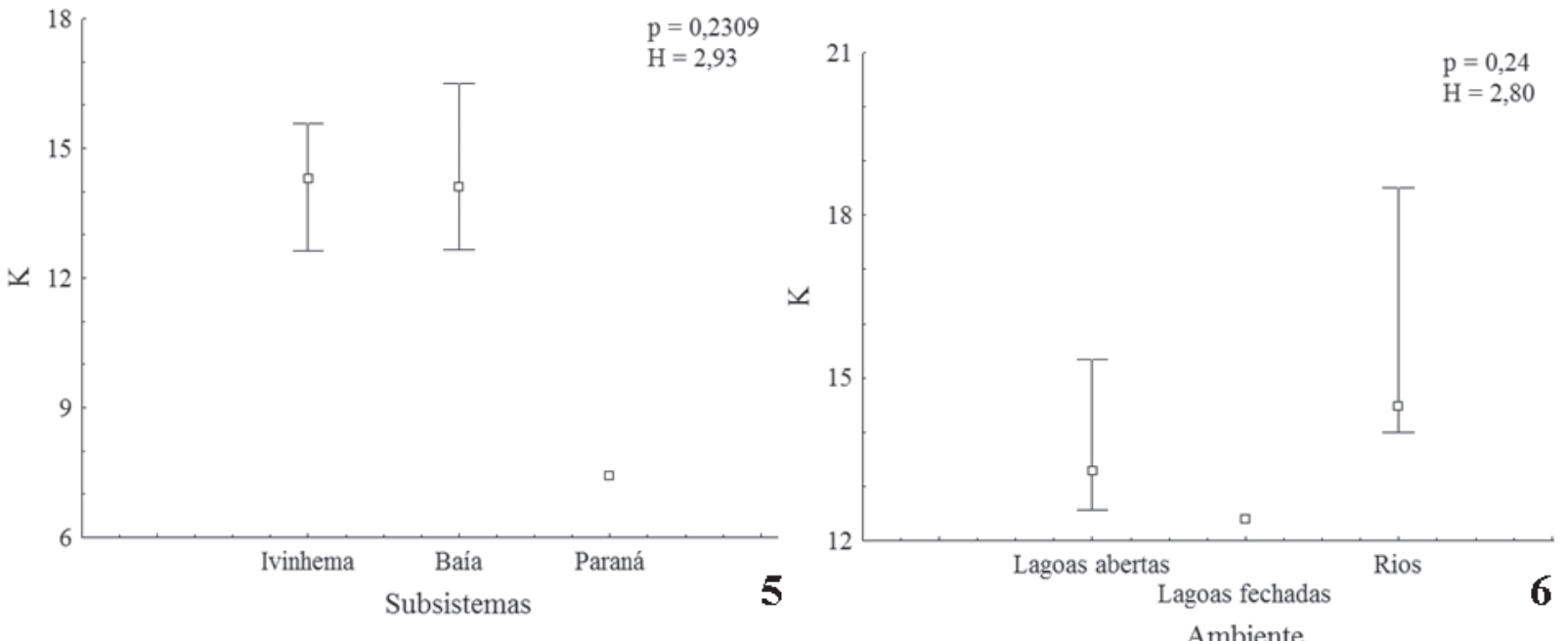

Figs 5, 6. Mediana ( $\square$ ) e percentis (barras verticais) do fator de condição por subsistema (5) e tipo de ambiente (6) para Pseudoplatystoma corruscans (Spix \& Agassiz, 1829) na planície de inundação do alto rio Paraná, entre junho de 2010 e março de 2011.
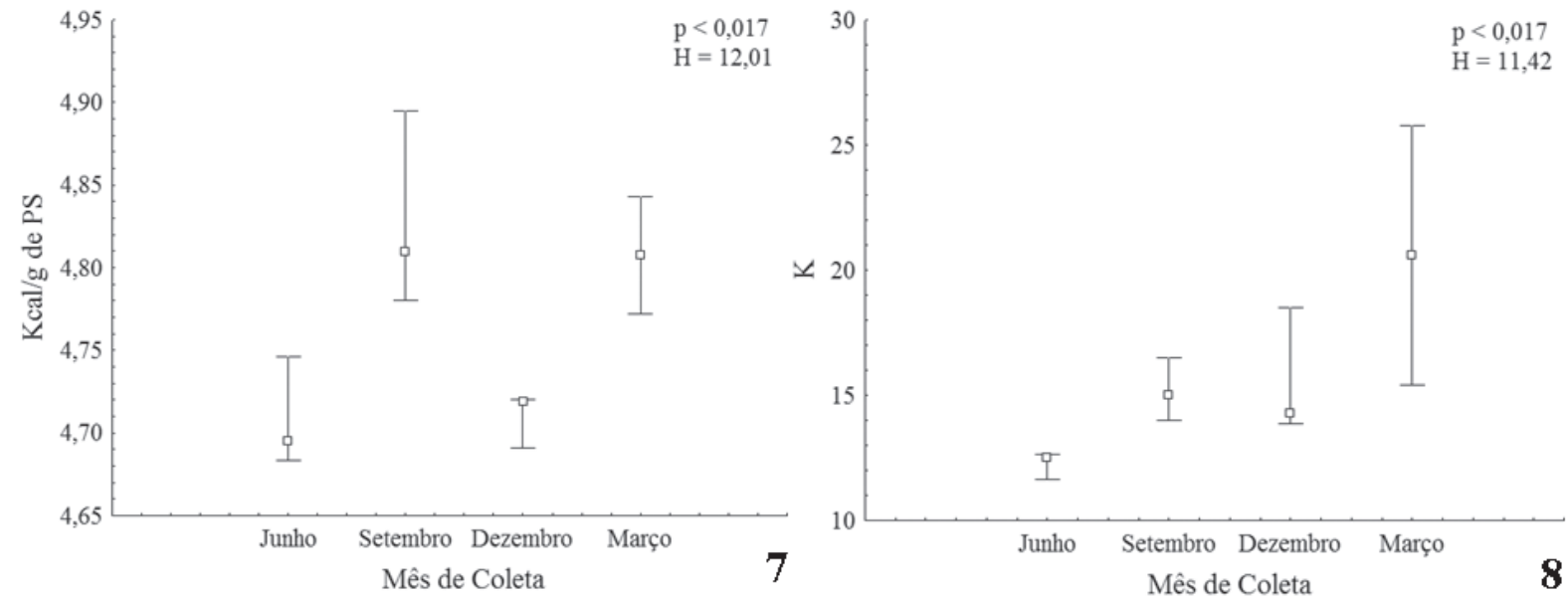

Figs 7, 8. Mediana ( $\square$ ) e percentis (barras verticais) da densidade energética (7) e do fator de condição (8) por subsistema mês de coleta para Pseudoplatystoma corruscans (Spix \& Agassiz, 1829) na planície de inundação do alto rio Paraná, entre junho de 2010 e março de 2011.
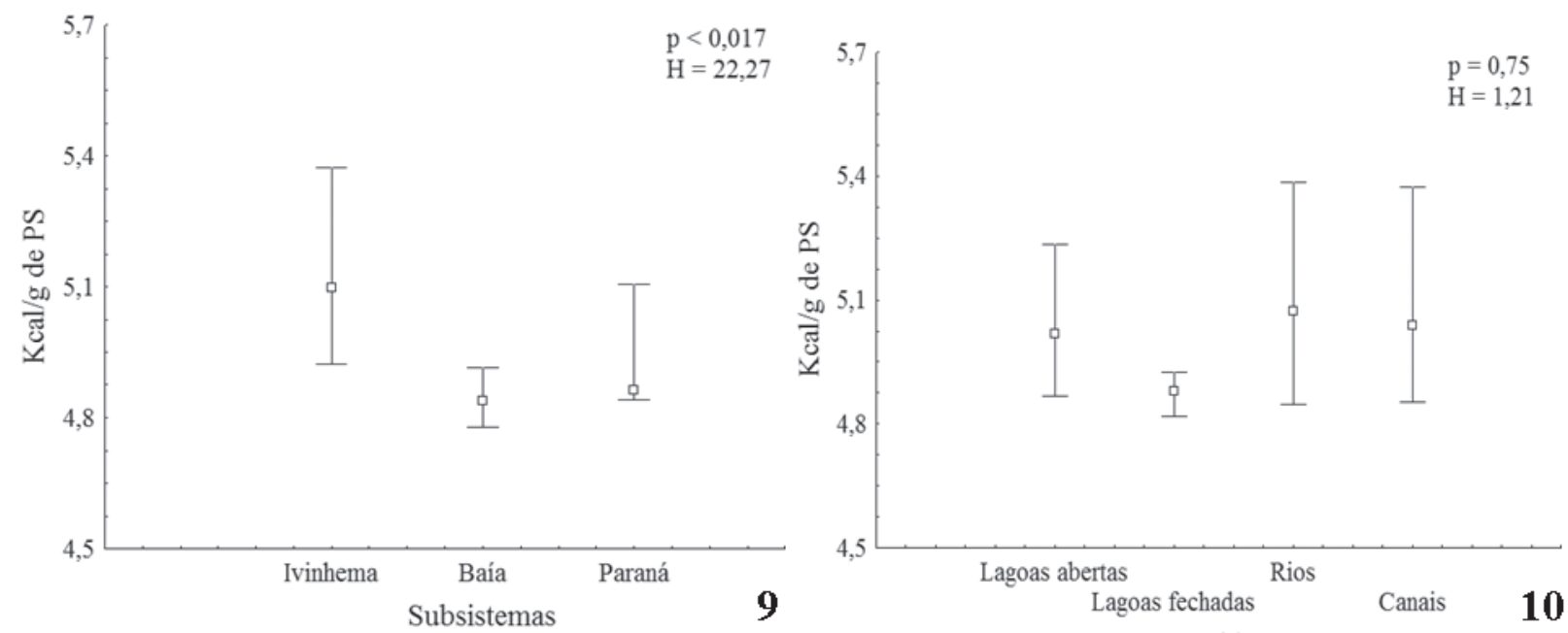

Figs 9, 10. Mediana ( $\square$ ) e percentis (barras verticais) da densidade energética por subsistema (9) e tipo de ambiente (10) para Prochilodus lineatus (Valenciennes, 1837) na planície de inundação do alto rio Paraná, entre junho de 2010 e março de 2011. 

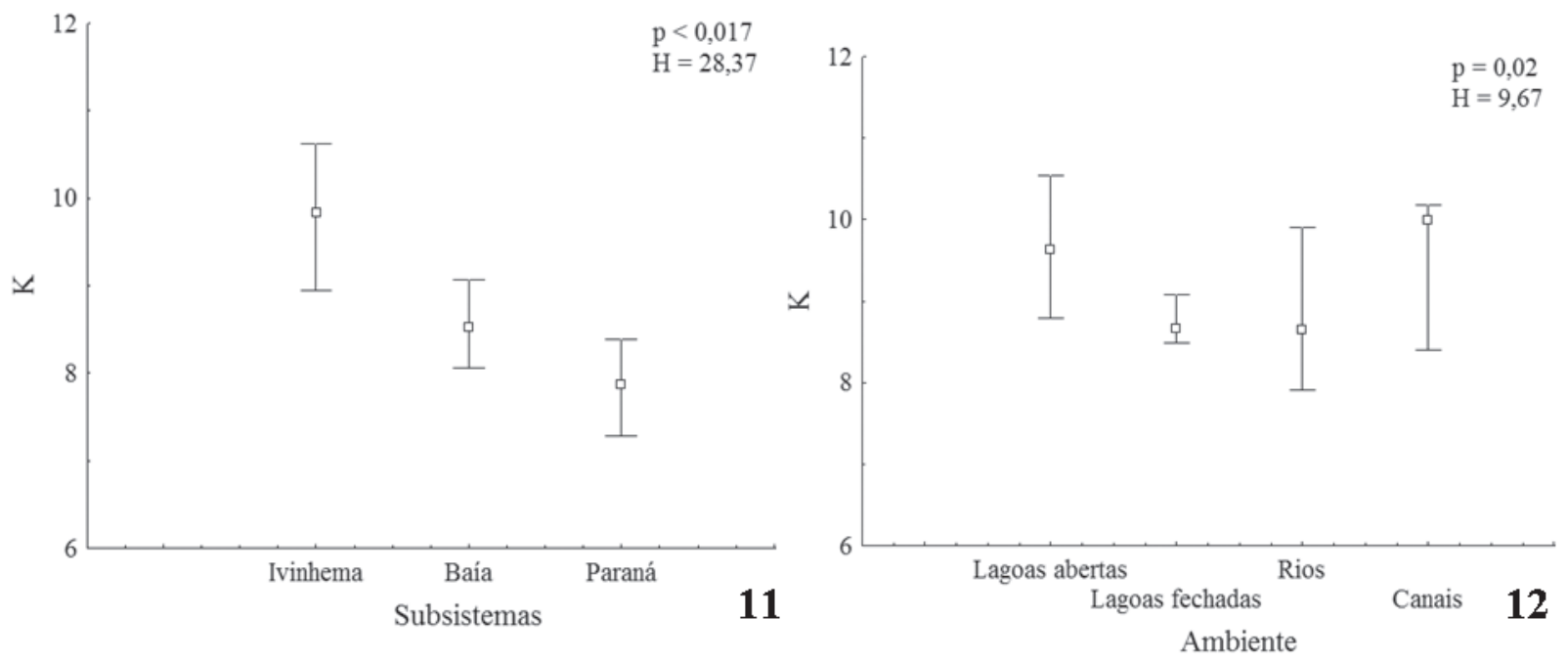

Figs 11, 12. Mediana ( $\square$ ) e percentis (barras verticais) do fator de condição por subsistema (11) e tipo de ambiente (12) para Prochilodus lineatus (Valenciennes, 1837) na planície de inundação do alto rio Paraná, entre junho de 2010 e março de 2011.
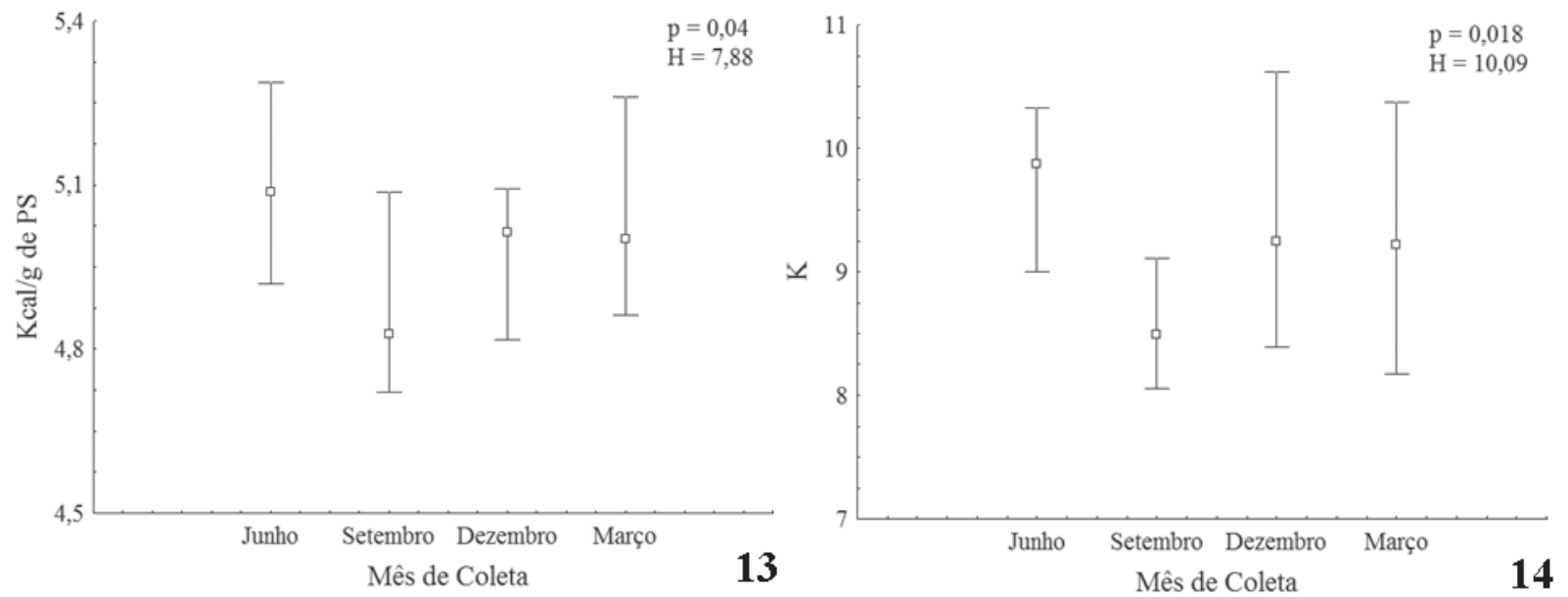

Figs 13, 14. Mediana ( $\square$ ) e percentis (barras verticais) da densidade energética (13) e fator de condição (14) por mês de coleta para Prochilodus lineatus (Valenciennes, 1837) na planície de inundação do alto rio Paraná, entre junho de 2010 e março de 2011.

no mesmo subsistema. Isso pode indicar que, entre os subsistemas amostrados, não há um que seja mais favorável para a espécie em termos de condições ambientais ou recursos alimentares. Além disso, a pequena quantidade de indivíduos capturados pode ter afetado os resultados das análises. Já para $P$. lineatus, diferente do que era esperado, a DE e o K mostraramse significativamente diferentes entre os subsistemas. Entretanto, observa-se que tanto a $\mathrm{DE}$ quanto o $\mathrm{K}$ apresentaram seus maiores valores no subsistema Ivinhema. Esse resultado pode ser explicado pelo fato deste abranger áreas de preservação ambiental (Parque Estadual do Ivinhema), onde o aporte de material alóctone é enriquecido por nutrientes, principalmente no período de inundação da planície, devido à rápida decomposição de gramíneas e restos de animais, ou ainda, pela camada humífera da floresta, o que leva a uma grande produção de detritos orgânicos, alimento preferencial de peixes iliófagos como P. lineatus (Lowe-McConNell, 1999; VISMARA et al., 2004). Estudos desenvolvidos em pequenos riachos e rios de grande porte (LowE-
McConnell, 1975; Goulding, 1980; ANGERmeier \& KARR, 1984), têm demonstrado que peixes de áreas tropicais dependem de recursos alimentares derivados diretamente da vegetação adjacente (UIEDA \& KIKUCHI, 1995). Assim, alterações de grande porte na composição e estrutura desta vegetação podem vir a causar sérios danos na integridade das comunidades aquáticas (ANGERMEIER \& KARR, 1984). Outros autores também discutem a importância do material alóctone para o ecossistema aquático e seu maior aporte em períodos de chuva ou de águas altas (Gregory et al., 1991; Garman, 1991; Teresa \& Casatti, 2010). Sendo assim, a maior contribuição de material alóctone pela área de preservação no entorno do subsistema Ivinhema, especialmente na época de águas altas, pode ter influenciado de forma decisiva a alimentação da espécie, com consequente aumento nos valores de DE e de K, em relação aos outros subsistemas.

Nas análises por tipo de ambiente, a ausência de diferenças significativas para $P$. corruscans pode ser resultado do baixo número de amostras obtidas em cada 
tipo de ambiente. Mas também não foram observadas diferenças significativas na $\mathrm{DE}$ de $P$. lineatus entre os ambientes, o que pode indicar que as espécies não manifestam preferência por locais específicos para obtenção de energia, já que são migradoras e fazem uso de toda a planície, de acordo com a disponibilidade de alimento. Apesar da não significância, os maiores valores de K para $P$. corruscans nos rios foi também discutido por VAZZOLER et al. (1997), que afirmam que esta espécie se alimenta preferencialmente nesse tipo de ambiente, onde também é mais capturada, provavelmente por estes locais proporcionarem uma maior quantidade e variedade de alimento para espécies piscívoras de grande porte, assim como as lagoas abertas, que mantém conexão com outros ambientes e permitem que as espécies possam nadar em busca de locais mais favoráveis ao seu desenvolvimento, principalmente no que se refere à alimentação (Agostinho et al., 1993; 2000). O mesmo pode ser afirmado em relação aos elevados valores de $\mathrm{K}$ de $P$. lineatus nos canais e lagoas abertas, habitats preferenciais da espécie, principalmente para os juvenis, que utilizam estes ambientes como locais de alimentação (AGOSTiNHO et al., 1993; 2004), proporcionando alocação energética para seu crescimento e desenvolvimento e refletindo em incremento nos valores de $\mathrm{K}$. Comparações feitas entre a DE e o K não resultaram em correlações significativas, resultado também observado por VISMARA et al. (2004) e SANTOS et al. (2006).

Os maiores valores de DE para P. corruscans foram verificados em setembro, indicando que os indivíduos estariam preparados para um novo período reprodutivo, resultado que, segundo Prus (1970), estaria relacionado ao período precedente à escassez do alimento, quando há diminuição da entrada de energia no hábitat, ou antes do período reprodutivo, quando as espécies, principalmente as migradoras, alocam boa parte de sua energia para utilizar durante a reprodução. De acordo com Graça \& Pavanelli (2007), o período reprodutivo de $P$. corruscans, na planície de inundação do alto rio Paraná, ocorre entre setembro e janeiro e, portanto, no presente estudo, eram esperados os maiores valores de DE para o período precedente (junho). Considerando que os picos de cheia ocorreram em março, o registro da alta DE em setembro indica um possível efeito tardio dos níveis fluviométricos sobre a atividade reprodutiva da espécie, e consequentemente, com reflexos na densidade energética da mesma. Os valores de $\mathrm{K}$ também exibiram diferenças significativas entre os meses de estudo, com os maiores valores registrados para março, constatandose uma tendência em acompanhar o padrão da DE.

Para a maioria dos grupos tróficos se espera um aumento na disponibilidade de alimentos durante a cheia, mas, para os piscívoros, isso não se aplica, pois, de acordo com Lolis \& ANDRIAN (1996), apesar de a quantidade de peixes ser alta durante a cheia, estes estão menos disponíveis à predação devido ao maior número de abrigos que o ambiente oferece. Assim, a recuperação da DE e do K para $P$. corruscans independe do período de cheia, o que é mostrado com seus altos valores em março, durante a enchente, e não após a mesma. Isso comprova que a quantidade de alimento disponível na planície, independente da fase do ciclo hidrológico, não limita o desenvolvimento da espécie.

Para P. lineatus é possível observar também alteração no seu ciclo reprodutivo, provavelmente influenciado pelo atraso da cheia, pois, segundo Graça \& Pavanelli (2007), a reprodução dessa espécie na planície de inundação do alto rio Paraná ocorre de outubro a abril e, no presente estudo, constatou-se que a reprodução, provavelmente, ocorreu entre junho e setembro, marcada pela forte redução dos valores de DE de um mês ao outro, ou seja, a espécie utilizou as reservas energéticas armazenadas para se reproduzir em um período anterior ao de costume. Quanto aos demais valores, os resultados mostraram-se semelhantes aos verificados para $P$. corruscans, com forte incremento na $\mathrm{DE}$ em março, evidenciando que, com a chegada da cheia, a espécie também já se encontrava energeticamente recuperada. Assim como para P. corruscans, o alimento não é fator limitante, mesmo sendo $P$. lineatus uma espécie iliófaga, a qual se beneficia das cheias, quando ocorre maior aporte de material alóctone para o ambiente aquático, garantindo uma alimentação farta para a espécie (LowEMCCONNELl, 1999).

Da mesma forma que o observado para $P$. corruscans, os valores de $\mathrm{K}$ de $P$. lineatus acompanharam o padrão de DE. Sendo assim, constata-se novamente que mesmo com a cheia tardia, os indivíduos já se encontravam energeticamente recuperados, fato confirmado pelos altos valores de $\mathrm{K}$ registrados em dezembro e março.

Embora a atividade reprodutiva das espécies não tenha sido investigada com detalhes, é notável que as modificações fisiológicas estão associadas ao pulso de inundação, o qual exerce grande influência sobre o ciclo de vida dos organismos, possibilitando que os peixes aproveitem as condições favoráveis dos períodos de águas altas, garantindo a sobrevivência da progênie, resultando em um sincronismo generalizado da reprodução com a subida do nível das águas (AgostinHo et al., 2004). No período de enchente na planície de inundação, ocorre uma expansão das áreas alagadas e de biótopos, fornecendo, além de alimento, abrigo aos juvenis. Distúrbios ambientais decorrentes de represamento têm provocado, nos últimos anos, alterações na intensidade e duração dos pulsos de inundação (AgostinHo et al., 2004), promovendo, mudanças comportamentais e adequações fisiológicas, como a alocação energética (VISMARA et al., 2004) e possíveis adaptações do ciclo reprodutivo.

Sendo assim, modificações sazonais na densidade energética de peixes não somente indicam alterações sazonais na condição corpórea, mas também podem estar associadas a alterações na biomassa da comunidade, abundância de alimento, além de mudanças climáticas (Bryan et al., 1996). Segundo Dourado \& BeneditoCeCILIo (2005), a energia contida nos músculos dos peixes 
pode ser reflexo da concentração de nutrientes presentes no ambiente, isto é, altos valores de DE podem indicar que o alimento não representa um fator limitante para as espécies, o que parece ser verdadeiro para a área estudada.

Similarmente, o bem estar geral do peixe pode sofrer forte influência da sazonalidade devido a fatores como reservas de gordura e peso das gônadas (SÁ et al., 2000). Neste contexto, estudos que acompanhem tais modificações merecem atenção particular, pois podem indicar o sucesso ou desaparecimento de uma espécie no ambiente, garantindo informações que conduzam à tomada de medidas, a fim de se obter proteção efetiva à biodiversidade.

Agradecimentos. As autoras agradecem ao Núcleo de Pesquisas em Limnologia, Ictiologia e Aquicultura (Nupelia) da UEM, pelo apoio logístico; ao $\mathrm{CNPq}$ pelo apoio financeiro e concessão de bolsas; aos companheiros do laboratório de Ecologia Energética, pelo auxílio na preparação das amostras.

\section{REFERÊNCIAS BIBLIOGRÁFICAS}

AbilhoA, V. \& Duboc, L. F. 2004. Livro Vermelho da Fauna Ameaçada no Estado do Paraná. Curitiba, Instituto Ambiental do Paraná (IAP). 630p.

Agência Nacional de Águas. 2011. Dados Hidrológicos da Estação Fluviométrica de Porto São José. Disponível em: $<$ http://hidroweb.ana.gov.br/>. Acesso em: 06.10.2011

Agostinho, A. A.; Gomes, L. C.; Verissimo, S. \& OKadA, E. K. 2004. Flood regime, dam regulation and fish in the Upper Parana River: efects on assemblage attributes, reproduction and recruitment. Reviews in Fish Biology and Fisheries 14:11-19.

Agostinho, A. A.; Thomaz, S. M.; Minte-Vera, C. V. \& Winemiller, K. O. 2000. Biodiversity in the high Paraná River floodplain. In: Gopal, B; JUnK, W. J. \& Davis, J. A. eds. Biodiversity in wetlands: assessment, function and conservation. Leiden, Backhuys Publishers. p. 89-118.

Agostinho, A. A.; Vazzoler, A. E. A. De M.; Gomes, L. C. \& OKADA, Е. K. 1993. Estratificación espacial y comportamiento de Prochilodus scrofa em distintas fases del ciclo de vida, em la planície de inundación del alto rio Paraná y embalse de Itaipu, Paraná, Brasil. Revue d'Hydrobiologie Tropicale 26(1):79-90.

ANGERmeier, P. L. \& KarR, J. R. 1984. Relationships between woody debris and fish habitat in a small warmwater stream. Transactions of the American Fisheries Society 113:716-726.

Azevedo, P. \& VieIRA, B. B. 1938. Biologia do saguirú (Characidae, Curimatinae). Memórias do Instituto Oswaldo Cruz 33(4):481553.

Bryan, S. D.; Soupir, C. A.; Duffy, W. G. \& Freiburger, C. E. 1996. Caloric densities of three predatory fishes and their prey in Lake Oahe, South Dakota. Journal of Freshwater Ecology 11(2): 153161.

Costa, R. S.; OKada, E. K.; Agostinho, A. A. \& Gomes, L. C. 2012. Variação temporal no rendimento e composição específica da pesca artesanal do Alto rio Paraná, PR - Brasil: os efeitos crônicos dos barramentos. Boletim do Instituto de Pesca 38(3):199-213.

Dourado, E. C. S. \& Benedito-Cecilio, E. 2005. Ecologia energética de peixes: influência de fatores abióticos e bióticos. Maringá, EDUEM. 53p.

Economids, P. S.; Pantis, J. \& Margaris, N. S. 1981. Caloric content in some freshwater and marine fishes from Greece. Cybium 5:97100

Fowler, H. W. 1950. Os peixes de água doce do Brasil. Arquivos de Zoologia do Estado de São Paulo 6(2):205-404

Fugi, R. \& HahN, N. S. 1991. Espectro alimentar e relações morfológicas com o aparelho digestivo de três espécies de peixes comedores de fundo do rio Paraná, Brasil. Revista Brasileira de Biologia 51(4):873-879.

Garman, G. C. 1991. Use of terrestrial arthropod prey by a streamdwelling cyprinid fish. Environmental Biology of Fishes 30:325331.

Goulding, M. 1980. The fishes and the forest: explorations in Amazonian Natural History. Berkeley, University of California Press. 279p.

Graça, W. \& Pavanelli, C. S. 2007. Peixes da planície de inundação do alto Rio Paraná e áreas adjacentes. Maringá, EDUEM. $241 \mathrm{p}$.

Gregory, S. V.; Swanson, F. J.; McKee, W. A. \& Cummins, K. W. 1991. An Ecosystem perspective of riparian zones. BioScience 41(8):540-551.

Junk, J. W.; Bayley, P. B. \& Sparks, R. E. 1989. The flood pulse concept in river floodplain systems. Canadian Special Publication of Fisheries and Aquatic Sciences 106:110-127.

Leite, R. G.; Barbieri, R. L. \& Hernandez-Blazquez, F. J. 1988. Morfologia do trato digestivo do Curimbatá, Prochilodus scrofa. Boletim do Instituto de Pesca 15(2):221-227.

Lolis, A. A. \& Andrian, I. F. 1996. Alimentação de Pimelodus maculates Lacépède 1803 (Siluriformes, Pimelodidae), na planície de inundação do Alto Rio Paraná, Brasil. Boletim do Instituto de Pesca 23:187-202.

Lowe-McConnell, R. H. 1975. Fish communities in tropical freshwaters. London, Longman. $337 \mathrm{p}$.

1999. Estudos ecológicos de comunidades de peixes tropicais. São Paulo, EDUSP. 453p.

NeIFF, J. J. 1990. Ideas para la interpretación ecológica del Paraná Interciência 15:424-441.

Prus, T. 1970. Calorific value of animals an element of bioenergetical investigations. Polish Archives of Hydrobiology 30:183-199.

Roberto, M. C.; Santana, N. F. \& Thomaz, S. M. 2009. Limnology in the Upper Paraná River floodplain: large-scale spatial and temporal patterns, and the influence of reservoirs. Brazilian Journal of Biology 69(2)(Suppl.):717-725.

SÁ, M. F. P.; BARBIERI, G. \& Verani, J. R. 2000. Analysis of Cyprinus carpio, Prochilodus cearensis and Colossoma macropomum behavior in policulture experiment, based on the condition factor Boletim do Instituto de Pesca 26(2):181-188.

SaldaÑa, J. \& Venables, B. 1983. Energy compartimentalization in a migratory fish, Prochilodus marie (Prochilodontidae), of the Orinoco river. Copeia 3:617-623.

Santos, M. H.; Benedito, E. \& Domingues, W. M. 2006. Efeito da maturação gonadal sobre a energia dos músculos de duas espécies de piranhas do reservatório do rio Manso, Estado do Mato Grosso. Acta Scientiarum, Biological Sciences 28(3):227-236.

Teresa, F. B. \& CASATTI, L. 2010. Importância da vegetação ripária degradada em região intensamente desmatada no sudeste do Brasil: um estudo com peixes de riacho. Pan-American Journal of Aquatic Sciences 5(3):444-453.

Thomaz, S. M.; Roberto, M. C. \& Bini, L. M. 1997. Caracterização limnológica dos ambientes aquáticos e influência dos níveis fluviométricos. In: VAzzoler, A. E. A. M.; Agostinho, A. A. \& Hahn, N. S. eds. A planície de Inundação do alto rio Paraná. Maringá, EDUEM. p.73-102.

Uieda, V. S. \& KiKUCHI, R. M. 1995. Entrada de material alóctone (detritos vegetais e invertebrados terrestres) num pequeno curso de água corrente na cuesta de Botucatu, São Paulo. Acta Limnologica Brasiliensia 7:105-114.

VAzzoler, A. E. A. M. 1996. Biologia da reprodução de peixes teleósteos: teoria e prática. Maringá, EDUEM. 169p.

Vazzoler, A. E. A. M.; Agostinho, A. A. \& Hahn, N. S. 1997. A planície de inundação do alto rio Paraná: aspectos físicos, biológicos e socioeconômicos. Maringá, EDUEM. 460p.

Vismara, M. R.; Benedito-Cecilio, E. \& Faria, A. C. E. A. 2004 Efeito da maturação gonadal sobre o conteúdo calórico e condição geral de peixes da planície de inundação do alto rio Paraná. Acta Scientiarum, Biological Sciences 26(2):1189-1199. 